\title{
The art of communicating: The Nuclear Cardiology Report
}

\author{
Frans J. Th. Wackers, MD, PhD, FASNC
}

See related article, pp. 858-868

One of the most difficult of human endeavors is adequate communication. In clinical medicine patient treatment decisions often depend on efficient communication and integration of multidisciplinary diagnostic findings.

Practicing physicians generally do not have in-depth knowledge of specialized diagnostic modalities and rely on communication of this information by specialists. It behooves the nuclear cardiologist to communicate to referring physicians with unequivocal, comprehensible and clinically useful reports, so that appropriate patient management decisions can be made. Until recently a physician's report was considered untouchable, a sacred private domain, not to be critiqued or regulated.

In this issue of the Journal Tilkemeier et $\mathrm{al}^{1}$ provide an analysis of observations in more than 1,300 US laboratories by reviewers of the Intersocietal Commission for Accreditation of Nuclear Laboratories (ICANL) about the quality of nuclear cardiology reports. Their analysis revealed that the majority $(57.2 \%)$ of laboratories applying for ICANL accreditation created reports that did not meet accreditation standards. Notably, more than one-third $(35.5 \%)$ of laboratories were non-compliant for important aspects of reporting. ICANL Standards require 18 predefined elements to be present in an optimal report. It is fair to acknowledge that not all

From the Section of Cardiovascular Medicine, Department of Internal Medicine and the Cardiovascular Nuclear Imaging and Stress Laboratories, Yale University School of Medicine, New Haven, CT.

Reprint requests: Frans J. Th. Wackers, MD, PhD, FASNC, The Section of Cardiovascular Medicine, Department of Internal Medicine and the Cardiovascular Nuclear Imaging and Stress Laboratories, Yale University School of Medicine, New Haven, CT 06510; frans.wackers@yale.edu.

J Nucl Cardiol 2011;18:833-5.

$1071-3581 / \$ 34.00$

Copyright $@ 2011$ American Society of Nuclear Cardiology.

doi:10.1007/s12350-011-9417-5
18 required elements appear equally important. Although some elements are required solely for the purpose of billing, others are indeed crucial for effective communication with referring physicians. Tilkemeier et al discuss each of the 18 elements in detail in their article. In my view, there are four aspects of a report that are essential for good communication: the final conclusion; the description of myocardial perfusion abnormalities; the integration of stress test results with image interpretation, and the use of clear English language.

The importance of nuclear cardiology reports has been the topic of discussion in the past. Cerqueira proposed in 1996 the "user-friendly report" emphasizing the necessity for clarity but also what should and what should not be in a nuclear report. ${ }^{2}$ In 2000 the ICANL was alarmed enough about the frequency of suboptimal reports that it published recommendations for standardization of the format and content of reports. ${ }^{3}$ It seemed clear that in addition to focusing on technical aspects of imaging, clinical applications and read-withthe-experts sessions, educational efforts at national meetings should also be directed towards the teaching of how to create good reports. Accordingly, since 2003 the ASNC Imaging Guidelines (and those of other professional societies) also include recommendations for reporting. ${ }^{4-6}$ The analysis by Tilkemeier et al indicates that suboptimal nuclear cardiology reporting at the present time is still a wide spread problem.

\section{THE FINAL CONCLUSION}

Obviously the most important part of a report is the final conclusion or impression. The conclusion must be stated in clear language. The conclusion is the part of a report that many physicians look at first. I feel strongly that the conclusion must contain the word "normal" or "abnormal", The words "probably" or "equivocal", should be, and can be, avoided most of the time. A referring physician is entitled to get a clear answer to his clinical question from the nuclear cardiology specialist and the report should of help in treating patients. Hesitation, vagaries, convoluted and qualifying statements in a Conclusion are not helpful. Conclusions like "No evidence of ischemia or scar', or other descriptions of what is not there, are needlessly unclear and confusing. 
In my own reports I like to summarize in a Conclusion the findings of the study as a concise clinical message to the referring clinician. For instance: "Abnormal stress/ rest attenuation-corrected SPECT imaging after good exercise showing a moderate sized area of anteroseptal ischemia." Rather than providing a list of numbered findings, e.g. 1. Good exercise; 2. Normal exercise ECG; 3. Moderate reversible anteroseptal defect; 4. etc...

Furthermore, the wording of the conclusion must be consistent with the rest of the report. Many laboratories now use computer-based templates or report generators that require computer fields to be filled-in or changed with patient-specific data. Unfortunately it is not rare that, due to negligence or failure to proofread before signing, contradictory language is found in the body of the report and Conclusion. For example, in a report of a patient who had pharmacological stress, the word "exercise" may appear out of the blue because a field of the template was not adjusted. Or, dipyridamole and adenosine are mentioned in the same report; or worse, a different anatomical location is mentioned in the body of the report than in the conclusion. Such mistakes may be just a nuisance but also may lead to confusion.

The interpreter, being the expert, also has to make a decision when discordant results are obtained, e.g., positive exercise ECG and normal perfusion images. Hedging, without a clear interpretative decision will leave the referring physician confused.

One of the most incongruous qualifiers that I have seen in reports is a final advice "that clinical correlation is recommended". This gratuitous statement is useless and dodges ultimate responsibility.

\section{DESCRIPTION OF FINDINGS}

Myocardial perfusion abnormalities should be described in terms of location, size, and reversibility. The location of a myocardial perfusion abnormality should preferably be described by combining areas that encompass coronary artery territories, rather than list a number of isolated anatomical segments. The nomenclature for tomographic imaging was standardized in 2002 and should be used exclusively. ${ }^{7}$ In some reports one may still encounter obsolete terminology, such as posterior wall, an anatomical area that no longer exists. It is also very important to communicate to the referring physician the degree of myocardial perfusion abnormalities. The extent of an anatomical abnormality may be described as, e.g., extending from apex to mid-ventricular, or from apex to base. Severity is usually described as small, moderate or large. Unfortunately, these terms are not defined and thus may mean different things to different people. Communication becomes worse when one uses terminology such as "mild to moderate" or "moderate to severe". The only thing that is clear that the test is not normal. It is long overdue that ASNC establishes a data bank that allows for standardization of categorization of the magnitude of myocardial perfusion abnormalities.

Most interpreting physicians have no problem with describing areas with decreased uptake. However, deciding whether decreased uptake is within normal variation or abnormal is more difficult and requires considerable, yet fallible, experience. Therefore, it is important that every interpreter of nuclear cardiology studies uses one of the commercially available and validated software packages that provide quantification of perfusion abnormalities against a normal database. Although not every software package computes exactly identical numbers for defect sizes, they do provide the reader with an unchanging reference and benchmark that enhances consistency and reproducibility of interpretation. Once again, an ASNC quantitative database would be useful to calibrate various software programs. For the referring physician such quantification would be helpful because of its consistency and it may enhance the understanding of nuclear cardiology reports.

\section{INTEGRATION OF STRESS AND IMAGING RESULTS}

In many laboratories one cardiologist performs stress testing and another nuclear cardiologist/nuclear physician performs image interpretation. Each physician creates a report. It is optimal, and required by the ICANL, that those two reports are completely integrated. This is particularly important when physical exercise is performed, but also for pharmacological stress. The nuclear cardiology report should start with a summary of the stress procedure (exercise or pharmacological protocol, stages completed and duration of exercise, METs achieved, dose of pharmacological stress agent, hemodynamic and ECG response and symptoms) and then followed by a description of imaging results. The stress information is important for appropriate interpretation of the overall stress imaging procedure. For instance, a relatively small myocardial perfusion defect has different clinical implications for a patient with excellent (e.g., $10 \mathrm{METs}$ ) exercise performance than for a patient with poor exercise capacity. In the latter patient one should be worried about inducible ischemia at low level of exercise and cardiac catheterization may be justified, whereas in the former patient, even though he/she may have coronary artery disease, prognosis is favorable and the patient may do well on optimal medical treatment alone. The level of exercise (poor, fair, good, excellent) should therefore always be mentioned in the conclusion of the report. Ischemic 
ECG changes during pharmacological stress suggest a high likelihood of significant coronary artery disease and therefore are relevant for the interpretation of the stress myocardial perfusion images. Abnormal blood pressure or heart rate responses, arrhythmias and any alarming symptoms must also be mentioned in the Conclusion of the fully integrated report. Radionuclide myocardial perfusion imaging is not only about the detection of perfusion defects. A study without myocardial perfusion defects, but with an enlarged dilated left ventricle with decreased ejection fraction, is not a normal study and should be reported as abnormal and cardiomyopathy.

\section{CLEAR LANGUAGE}

Curiously, some people who speak perfectly normal and understandable English in normal life may use very strange, indirect and convoluted language when creating a nuclear cardiology report. One of the reasons for unclear and cautious language may be the hesitation to commit unequivocally to either normal or abnormal interpretation. Not infrequently an interpreter has to make a choice between artifact and true perfusion abnormality. The interpreter, being the expert, should not share his/her ambiguity with the referring clinician, but bite the bullet and commit him/herself one way or the other in clear language. The interpreter of stress nuclear cardiology studies should always aim to provide a clear and understandable answer to a clinical question. The interpreter should also be aware that unclear communication may have unintended consequences for the patient. In complicated cases it is preferable to pick up the phone and communicate and discuss directly with the referring physician.

Tilkemeier et al showed that $23.6 \%$ of reports did not have integrated stress and imaging results and that in $4.5 \%$ the impressions were not considered "succinct". 1 There were interesting geographical differences between East and West Coast that are not well explained. One may feel that accreditation is just one more burden imposed on physicians and laboratories in an already (over) regulated medical practice environment. However, Tilkemeier's analysis suggests that laboratories that have gone through multiple accreditation cycles are more likely to be compliant with ICANL standards and produce better quality nuclear cardiology reports than laboratories that apply for the first time. Thus, the process of accreditation, i.e., submitting data on all aspects of a laboratory and receiving feedback from reviewers, appears to have educational value.

As a reviewer for ICANL I have had discussions and disagreements on how to deal with English style and wording in reports. There was always a reluctance to interfere with such a personal thing as a physician's words in a report. Obviously, appreciation of style is subjective. And, not being a native English speaker myself, I may be on shaky grounds. However, the report, being an instrument of communication, must be evaluated for its effectiveness. Nuclear cardiology is not that esoteric that it cannot be described in plain English. If the wording of a report does not convey a clear message that is informative to the referring physician, communication has failed, as well as the purpose of performing the test in the first place.

\section{Conflict of interest}

Frans J. Th. Wackers, MD is the founder and a past president of the ICANL and currently a reviewer for the ICANL. The author has indicated no financial conflicts of interest.

\section{References}

1. Tilkemeier P, Serber E, Farrell MB. The nuclear cardiology report: Problems, predictors and improvement. A report from the ICANL database. J Nucl Cardiol 2011. doi:10.1007/s12350-011-9390-z.

2. Cerqueira MD. The user-friendly nuclear cardiology report: What needs to be considered and what is included. J Nucl Cardiol 1996;3:350-5.

3. Wackers FJTh. Intersocietal Commission for the Accreditation of Nuclear Medicine Laboratories (ICANL) position statement on standardization and optimization of nuclear cardiology reports. J Nucl Cardiol 2000;7:397-400.

4. Hendel RC, Wackers FJTh, Berman DS, et al. Reporting of radionuclide myocardial perfusion imaging studies. J Nucl Cardiol 2003; $10: 705-8$

5. Imaging Guidelines for Nuclear Cardiology Procedures, 2009. Standardized reporting of radionuclide myocardial perfusion and function. http://www.asnc.org/section_73.cfm. Accessed May 2011.

6. ACCF/ACR/AHA/ASE/ASNC/HRS/NASC/RSNA/SAIP/SCAI/ SCCT/SCMR. 2008 Health policy statement on structured reporting in cardiovascular imaging. J Am Coll Cardiol 2009;53:76-90.

7. Cerqueira MD, Weisssman NJ, Dilsizian V, et al. Standardized myocardial segmentation and nomenclature for tomographic imaging of the heart: a statement for healthcare professionals from the Cardiac Imaging Committees of the Council on Clinical Cardiology of the American Heart Association. Circulation 2002;105:539-42. 